\title{
LA NECESIDAD DE CONOCIMIENTO Y RECONOCIMIENTO PLURAL. LOS PUEBLOS INDÍGENAS EN LA PROVINCIA DE BUENOS AIRES, ARGENTINA
}

CAROLINA MAIDANA*

En Argentina -como en el resto de América Latina y en el mundo- la presencia de los pueblos indígenas y sus formas organizativas se hace cada vez más visible. Producto de sus luchas, actualmente estos son reconocidos incluso a partir de herramientas censales $y$ marcos legales instituidos.

En cuanto a las estimaciones demográficas en Argentina, cabe señalar que en los tres primeros censos nacionales -realizados en 1869, 1895 y 1914- la temática indígena tuvo un tratamiento parcial, mientras que en los realizados en los años subsiguientes -1947, 1960, 1970,1980 y $1991-$ directamente no se incluyó la variable indígena en las mediciones ${ }^{1}$. Esta fue incorporada -según lo establecido por la Ley Nacional 24956/98- en el Censo Nacional de Población, Hogares y Viviendas de 2001, que marcó, por ello, un hito en la historia demográfica del país (INDEC, 2006). En él se estimó la cantidad de hogares del país donde al menos una persona se reconocía como perteneciente o descendiente de algún pueblo indígena. Luego -de acuerdo a lo instituido en el Artículo 75, Inciso 17 de la Constitución Nacional- se elaboró una Encuesta Complementaria de Pueblos Indígenas (ECPI) 2004-2005, con la cual, desde las distintas direcciones provin- ciales de estadística, se retornó a una parte de los hogares detectados a través del Censo 2001 con el objetivo de caracterizar al conjunto de la población que integraba estos hogares.

En el último Censo Nacional, llevado a cabo por el Instituto Nacional de Estadísticas y Censos (INDEC) en el año 2010, en la pregunta por el autorreconocimiento se consideró no solo la cantidad de hogares, sino también la cantidad de personas que se reconocían como miembros/ descendientes de un pueblo indígena. Esto fue evaluado por algunos referentes indígenas como una mejora respecto del Censo de 2001, en cuanto posibilidad de contribuir a visibilizar sus colectivos de pertenencia, incrementando así sus fuerzas y resistencias, en el sentido de habilitar demandas y expandir derechos (Maidana, Tamagno \& Martínez, 2014).

En esa oportunidad se contabilizó, a nivel nacional, la presencia de 955.032 personas pertenecientes o descendientes de alguno de los 31 pueblos indígenas reconocidos por esta herramienta censal ${ }^{2}$, lo cual representa $2,38 \%$ del total de la población del país. En la provincia de Buenos Aires, en la Región Metropolitana ${ }^{3}$, se registró la presencia de 248.516 personas que se reconocen como pertenecientes 0

\footnotetext{
* Antropóloga - Dra. en Ciencias Naturales. Investigadora del Consejo Nacional de Investigaciones Científicas y Técnicas (CONICET) y docente de la Universidad Nacional de La Plata (UNLP) y la Universidad Nacional de Quilmes (UNQ).
} 
descendientes de pueblos indígenas, cifra que representa 1,9\% del total de la población de la región. Por su lado, en el interior de la provincia se contabilizaron 112.671 personas que se reconocen indígenas ( $2 \%$ de la población total del interior de la provincia).

En cuanto a las comunidades indígenas de la provincia de Buenos Aires, en el listado publicado por el Instituto Nacional de Asuntos Indígenas (INAI) a fines del año $2018^{4}$ se señala la presencia de 52 comunidades indígenas con personería jurídica registrada ${ }^{5}$ o con relevamiento técnico, jurídico y catastral a través del Programa Nacional Relevamiento Territorial de Comunidades Indígenas (Re.Te.C.I.) ${ }^{6}$, ya sea que esté en trámite o culminado y que no hayan registrado la personería.

Los registros de los propios referentes indígenas, las producciones científicas sobre la temática y las publicaciones/informes de distintos organismos no gubernamentales (ONG) señalan subregistros en los censos y las estadísticas oficiales debido a la imposición de diversas categorías y criterios de identificación, lógicas clasificatorias y modos de implementación de las herramientas censales. Sin embargo, y más allá de esta limitación concreta, las cifras arrojadas dan cuenta de la significación de la presencia indígena en un territorio donde la misma fue sistemáticamente negada. Esto explica que las valoraciones positivas realizadas por referentes indígenas respecto a estas estimaciones se relacionen a las posibilidades de conocer "donde estamos", ya que según la propia gente "muchas veces cuando llegamos a un organismo gubernamental con un reclamo, los funcionarios desconocen que aquí o allí vivimos indígenas" (Maidana, Zubrzycki \& Ottenheimer, 2014).
Al desconocimiento del lugar donde se encuentran personas y comunidades de los distintos pueblos indígenas que habitan el país se suma, con frecuencia, la ignorancia de las leyes que los asisten en cuanto colectivos culturalmente diferenciados. Sin embargo, la vulneración sistemática de los derechos de los pueblos indígenas no solo responde a estas inadvertencias, sino que también expresa las grandes dificultades encontradas para la reglamentación y la aplicación de las normativas. Es en este sentido que la presencia de estos pueblos y sus demandas constituyen un notable desafío para los organismos de gobierno que deben responder a las mismas.

En este contexto, y a partir de reclamos concretos de referentes de los pueblos indígenas que habitan el territorio bonaerense ${ }^{7}$, la Defensoría del Pueblo de la Provincia de Buenos Aires ${ }^{8}$ solicitó asesoramiento técnico al Laboratorio de Investigaciones en Antropología Social $\left(\right.$ LIAS) ${ }^{9}$ a los fines de contar con elementos teórico-académicos que medien en la resolución de dichas demandas. Debido a nuestro posicionamiento teórico-epistemológico convocamos, entonces, a referentes de la Comunidad Nam Qom, con quienes compartimos la trayectoria de investigación para dar respuesta a este requerimiento ${ }^{10}$.

Fue así que en 2017 se generó un "Protocolo de actuación para organismos gubernamentales de la provincia de Buenos Aires que reciben demandas de personas, comunidades y pueblos indígenas por la efectivización de sus derechos"11, a los fines de contribuir tanto al reconocimiento pleno de la existencia de los pueblos indígenas que habitan el territorio provincial y nacional como a hacer efectivo el cumplimiento de los derechos de estos pueblos 
y sus integrantes. Este protocolo fue diseñado en el marco de un protocolo adicional al Convenio de Cooperación Técnica y Asistencia Recíproca celebrado entre el defensor del pueblo de la Provincia de Buenos Aires y la Universidad Nacional de La Plata (UNLP)-Facultad de Ciencias Naturales y Museo (FCNyM), que forma parte de las políticas de defensa y promoción de los derechos humanos que la Defensoría del Pueblo de la provincia de Buenos Aires lleva adelante. Estas políticas implican la colaboración con instituciones académicas sobre temas de derechos humanos no solo con la finalidad de promover su reconocimiento y respeto, sino también -y fundamentalmentecon el objetivo de aportar elementos útiles a quienes deben responder a las demandas sobre su cumplimiento y garantizarlos.

El Protocolo recupera cifras censales, una síntesis del marco jurídico referido a pueblos indígenas y una serie de recomendaciones que son el resultado de una construcción colectiva, de una producción de conocimiento conjunto (Tamagno et al., 2005) en el marco de una investigación de larga duración (Braudel, 1970 [1968]) desarrollada desde el Laboratorio de Investigaciones en Antropología Social (LIAS) en diálogo tanto con referentes y organizaciones indígenas como con el conocimiento antropológico producido en América Latina sobre los pueblos a los que estos referentes y organizaciones pertenecen. En este sentido, constituye una herramienta-guía para orientar a los funcionarios públicos en la atención de las demandas y las necesidades de los pueblos indígenas y las personas y las comunidades que los integran. Las recomendaciones allí brindadas han sido redactadas teniendo en cuenta los siguientes objetivos:
- Contribuir al conocimiento de la legislación sobre pueblos indígenas y evitar su incumplimiento.

- Contribuir a saldar la distancia entre lo reconocido en las normas jurídicas que garantizan los derechos indígenas y la reglamentación y aplicación de las mismas por parte de los organismos gubernamentales.

- Contribuir a superar las posibles distancias entre verdad legal o judicial y verdad real ${ }^{12}$.

Las consideraciones que allí se presentan producto, como señalamos anteriormente, de una investigación de larga duración fundada en el diálogo con un sinnúmero de referentes indígenas y con el conocimiento antropológico sobre los pueblos a los que estos referentes pertenecen- ponen el acento en los procesos de transformación y cambio sin pérdida de identidad, al fundarse en el análisis de aquellos nucleamientos/asentamientos/barrios/comunidades cuyos referentes y líderes políticos y/o religiosos se reconocen como parte de las transformaciones y las trayectorias de pueblos indígenas que no han desaparecido. En este sentido, los principios guía para la acción-recomendaciones allí realizados refieren a:

1. La diversidad cultural y la autoadscripción. Se señala que el diseño de toda política indígena debe partir de revisar la conceptualización de la Argentina como país "blanco" y sin "indios" que impide reconocer en toda su dimensión las trayectorias de los pueblos indígenas y su situación actual. En este contexto, se recuperan cifras censales y se indica la centralidad del principio de autoadscripción recordando que no corresponde a los funcionarios de organismos gubernamentales ni a los especialistas 
esgrimirse como "censores de indianidad" (Tamagno, 1991), sino que es facultad de las personas y las comunidades definirse como parte de estos pueblos, tal como lo establece el Convenio 169 de la Organización Internacional del Trabajo (OIT) ${ }^{13}$.

2. La libre determinación. Se indica -con base en la Declaración de las Naciones Unidas sobre los Derechos de los Pueblos Indígenas (aprobada por Argentina en 2007)- la necesidad de reconocer todas las formas organizativas que se den a sí mismos estos pueblos y, en ese sentido, que el Estado-nación se identifique como plurinacional.

3. Los liderazgos indígenas. Se plantea que los líderes deben ser considerados referentes en cuanto expresan, a nivel individual, las transformaciones, las luchas y las demandas de los colectivos con los cuales se referencian.

4. La consulta y el consentimiento libre, previo $e$ informado. Se recuerda que es obligación de los Estados consultar a los pueblos indígenas sobre asuntos que los afecten en distintos contextos.

5. El acceso pleno al sistema de justicia del Estado nacional. Se indica que en todo procedimiento judicial se deben considerar las visiones de mundo de estos pueblos.

6. Las transformaciones y las continuidades en las presencias indígenas. Se señala que al abordar la cuestión territorial es imprescindible considerar el etnocidio $y$, al mismo tiempo, el hecho de que las culturas no mueren, sino que se transforman, y que estos procesos implicaron también movilidad geográfica.
7. Su carácter de sujetos sociales. Se señala que es imprescindible preguntarse sobre quiénes produjeron las acciones que derivaron en las situaciones en que hoy se encuentran los pueblos indígenas, dejando en claro que las mismas son producto de la explotación y la desigualdad y no de condiciones internas de dichos conjuntos.

8. Las lógicas comunitarias. Se indica que lo comunitario no se limita a la territorialidad y tampoco implica necesariamente hablar una lengua particular o compartir una religión: es sentirse parte de un conjunto, por lo cual sus derechos deben ser comprendidos en su carácter de derechos colectivos, a pesar de que la "lógica jurídica", fundada en una concepción individualista, dificulta la efectivización de estos "derechos colectivos", que solo tienen cabida en el marco de la persona o personería jurídica.

9. El concepto de pueblos indígenas. Se explica que el sujeto de derecho es el pueblo indígena y que el reconocimiento de las personerías jurídicas a sus comunidades es consecuencia de esta afirmación.

10. La historización de las demandas / tensiones / resistencias. Se indica que los acontecimientos que puntualmente generan demandas deben ser comprendidos en la dimensión histórica que los explica.

11. El racismo presente en el sentido común. Se señala que no debemos perder de vista que el racismo aparece ante los intereses de los sectores dominantes y sus proyectos en el contexto de la sociedad de mercado.

12. Las políticas indígenas. Se concluye que las políticas culturales no deben desvincularse 
de las demandas sobre la tierra, la salud, la educación, el trabajo y vivienda dignos.

Sobre este último punto -que llama la atención respecto de las políticas indígenas que con frecuencia apelan solo a lo cultural relegando las luchas contra la exclusión, la inequidad socioeconómica y el racismo- se han manifestado un sinnúmero de veces los referentes indígenas con quienes dialogamos. En el marco del V Congreso Argentino de Antropología Social ${ }^{14}$, realizado en la ciudad de La Plata entre el 29 de julio y $1^{\circ}$ de agosto de 1997, un referente indígena toba (qom) expresaba:

Sepamos todos que la frase que ustedes hacen: "EI indio es el dueño de la tierra", perdone que les diga que, entonces, ustedes también son indios. Pero, yo quisiera no tomarme en esa frase, sino que aquí, en este momento, en esta altura, en esta etapa, yo quisiera llegar a una nueva: que nos hermanamos todos y que nos sentimos en el mismo sentir.

Queremos que haya una transformación de la parte nuestra. No que viene uno y dice: "Yo respeto a tu cultura, yo respeto a sus costumbres". Como diciéndome: "viví como podés vivir", "quedate como estás viviendo".

No, me dicen: "hay que vivir tal cosa"; no, me dice: "busquen la forma de transformarse"; no: "yo la respeto a tu cultura, a tu forma de vivir" [...] y sigue siendo pidiendo limosna, porque sigue siendo dando limosna.

Llamaba así la atención sobre la necesidad de colaboración intercultural, sobre el valor político y humanístico de la investigación etnográfica capaz de contribuir al reconocimiento pleno de los pueblos indígenas que configuran sociedades pluriculturales y multiétnicas. En palabras de Miguel Bartolomé (2003):

(...) nos encontramos en un momento donde lo que se requiere es no sólo entender y criticar los acontecimientos actuales, sino también contribuir a imaginar el futuro repensando el presente y haciendo ver, a partir del cuestionamiento de esa construcción arbitraria que llamamos realidad, la pluralidad de realidades posibles. $Y$ una de esas alternativas está representada por la configuración de formaciones estatales culturalmente plurales, donde las poblaciones indígenas tengan acceso a los recursos económicos, políticos y culturales que posibiliten su reproducción y su futuro (p. 2001).

\section{El marco teórico/epistemológico que guió la redacción del Protocolo: sobre el "conocimiento plural"}

En 2017, en el marco de la XII Reunión de Antropología del MERCOSUR ${ }^{15}$, realizada en la ciudad de Posadas entre el 4 y el 7 de diciembre, una referente indígena del pueblo avá guaraní expresaba:

En este tiempo de la historia, de haber ingresado al siglo XXI con todo el adelanto de la ciencia, cuando se pregona fuertemente la democracia y el respeto a los derechos humanos, aún seguimos sufriendo el continuo despojo de la tierra y la esclavitud aún persiste, bajo diferentes formas, desde el lento proceso de colonización que se inició con la conquista [...] Con respecto a la política de los gobiernos, es una política desalentadora y humillante para nosotros, son solo políticas paternalistas, en donde se considera al indio imposibilitado de desarrollarse por sus propios medios [...] Para poder defender nuestros derechos y expresar nuestras necesidades los pueblos originarios recurrimos a distintas formas de organización y movilización social que, con frecuencia, resulta ser la única manera de hacer que nuestras demandas sean escuchadas. La protesta social es criminalizada, lo que da lugar a nuevas $\mathrm{y}$, a veces, serias violaciones a los derechos humanos. Muchos de estos incidentes ocurren cuando las organizaciones y comunidades indígenas intentamos defender la tierra, recursos naturales y territorios ancestrales. El aumento de las migraciones es otra de las expresiones de la globalización, de la desigualdad y de la pobreza generada por esta [...] La migración forzada de los pueblos indígenas es el resultado de la situación, muchas veces desesperada, que experimentamos en nuestros lugares de origen. 
De esta forma daba cuenta de las situaciones de tensión, violencia y represión que acusan los pueblos indígenas, frente a las cuales resulta indispensable pensar en términos de etnicidad -definida por A. Cohen (1974) como la forma de interacción entre grupos culturales operando dentro de contextos sociales comunes-. Como antropólogas y antropólogos enfrentamos hoy este desafío que implica repensar los conceptos de interculturalidad y comunidad, dos nociones que vertebran las políticas indígenas nacionales de los últimos treinta años -Ley 23.302 (B.O 12 de noviembre de 1985), Art. 75 Inc. 17 de la Constitución Nacional reformada en 1994 y Ley 26.160 (B.O. 29 de noviembre de 2006) ${ }^{16}$. Su definición representa un reto tanto para que los pueblos indígenas puedan gozar de los derechos que estas normativas contemplan como para que los entes gubernamentales que deben aplicarlas puedan dar respuestas satisfactorias en este sentido, siendo uno de ellos la Defensoría del Pueblo de la provincia de Buenos Aires.

Con la realización del "Protocolo de actuación para organismos gubernamentales de la provincia de Buenos Aires que reciben demandas de personas, comunidades $y$ pueblos indígenas por la efectivización de sus derechos", los avances de la línea de investigación "Identidad/etnicidad/interculturalidad. Indígenas en ciudad", desarrollada desde el LIAS, trascendieron, una vez más, los espacios puramente académicos para aportar a la configuración de un conocimiento de valor para el tratamiento de la cuestión indígena.

La preexistencia de los pueblos indígenas respecto del Estado argentino, reconocida en el Artículo 75, Inciso 17 de la Constitución Nacional, requiere de un tratamiento particular en cuanto remite a la diversidad cultural que debe ser entendida en su relación con aspectos de orden económico y político. Rodolfo Stavenhagen (2007), quien fuera relator especial de las Naciones Unidas sobre la Situación de los Derechos Humanos y Libertades Fundamentales de los Pueblos Indígenas, plantea:

En diversas esferas hay una incomprensión con respecto a los derechos indígenas, ligada a la persistencia de prejuicios y actitudes discriminatorias cuando no racistas. Más preocupante resulta la oposición al pleno disfrute de sus derechos que proviene de diversos intereses económicos privados, nacionales e internacionales. Estos intereses se centran en la propiedad de la tierra y la explotación de los recursos naturales, sobre todo los bosques, el agua y los recursos del subsuelo. Con frecuencia están coludidos con las estructuras del poder político para obstaculizar el avance de los derechos humanos de los pueblos indígenas (p. 21).

Partiendo de este postulado, el desarrollo de nuestra línea de investigación se ha fundado tanto en la construcción de lineamientos de análisis teóricos como en la construcción de lineamientos de análisis epistemológicos.

Cuando remitimos a los lineamientos de análisis teóricos nos referimos a aquellas proposiciones que figuran en nuestra producción académica y que han sido construidas en la dialéctica teoría y práctica que caracteriza todo proceso de investigación. En dicho proceso nos hemos enriquecido en el diálogo con propuestas investigativas que no soslayan el análisis de la estructura social y, por lo tanto, no obvian la subsunción de las problemáticas analizadas en los contextos de tensión que se caracterizan por significativas desigualdades sociales. La etnicidad es la expresión política de la identidad étnica, y esta última se dinamiza y recrea constantemente en función de contextos carac- 
terizados por tensiones de poder y desigualdad, por lo que se hace imposible comprenderla cabalmente si las cuestiones estructurales no son tenidas en cuenta.

Estos planteos se han traducido en un particular modo de hacer ciencia, de hacer antropología, que condujo a que se nos requiera como consultores o asesores tanto por parte de organismos gubernamentales y no gubernamentales que tienen como objetivo la planificación y puesta en práctica de políticas sociales como por parte de conjuntos sociales que acuden al saber académico como un modo de legitimar sus presencias y demandas. Cabe aclarar que si bien en algunos casos hemos sido convocados formalmente y hemos podido dar cuenta de una actividad explícitamente reconocida por el ámbito universitario (por ejemplo, bajo la forma de servicios a terceros) son innúmeros los casos en que hemos respondido a requerimientos sin formalismo alguno, excepto el de colocar las preocupaciones y las demandas que se nos planteaban en el contexto de la dinámica de las propias investigaciones.

Cuando aludimos a la construcción de lineamientos de análisis epistemológicos nos referimos a algo que se vincula con lo antedicho y que nos permite avanzar en una serie de aseveraciones sobre los modos de producir conocimiento. Estos se vinculan directamente con la valoración y con el análisis del conocimiento que se produce en la relación dialéctica entre investigadores y sujetos que forman parte de los objetos de investigación. Se fundan en la comprensión de que si bien el conocimiento tiende hacia la verdad absoluta, lo que producimos son verdades parciales, a las que se les pueden hacer nuevas preguntas en función de nuevos modos de abordaje o de las transformaciones propias de las dinámicas de los objetos de análisis. Siempre construimos conocimiento con el "otro", el "otro" al que referenciamos académicamente y el "otro" al que interrogamos con nuestras inquietudes, generando, al mismo tiempo, la posibilidad de que se interrogue a sí mismo.

En este sentido, el desarrollo de nuestra investigación implicó, desde sus orígenes, y en el marco de lo que Bourdieu, Chamboredon \& Passeron (1975) definen como objetivación participante, la valoración del diálogo con los referentes indígenas junto a quienes trabajamos. Fue con frecuencia en el intercambio de pareceres donde se manifestaron aspectos que serían decisivos en la producción de conocimiento. La articulación entre investigación y extensión universitaria generó que una de las preguntas clave con las que comenzamos la trayectoria de investigación fuera "¿qué es lo que quisieran contar de ustedes mismos?"17.

En ese marco, acuñamos el término de "producción de conocimiento conjunto" (Tamagno et al., 2005), por medio del cual reconocíamos la significación del saber aportado por los referentes indígenas a la reflexión crítica. Sin estos saberes, el conocimiento producido corre el riesgo de estar sustentado en prejuicios o en apreciaciones de sentido común que conducen a lo que hemos denominado críticamente actitudes propias de los "censores de la indianidad" (Tamagno, 1991). Desde este posicionamiento organizamos, a lo largo del tiempo, actividades en congresos, jornadas y talleres donde referentes indígenas de distintos pueblos expresaron las preocupaciones y las demandas que vertebran las luchas de los colectivos a los que pertenecen y que generan sus formas organizativas. También reflexionaron sobre las 
maneras en que estas han interpelado e interpelan la labor antropológica ${ }^{18}$.

Ante la sensación de que todavía se investiga "sobre" y no "con" o "junto a" hemos buscado trascender el denominado "populismo metodológico", producto del cual los reconocimientos respecto de la dialéctica antropología-pueblos indígenas quedan a nivel discursivo y no se traducen en prácticas concretas (Lima, 2012). Hemos trabajado desde un enfoque interepistémico, es decir, que parte de la premisa de que no hay saber universal, sino que existen muchas configuraciones del conocimiento vinculadas a diversas condiciones de producción y, por lo tanto, que el intercambio y la colaboración entre diferentes formas de saber son imprescindibles (Escobar, 2014; Mato, 2008). Es el diálogo intercultural lo que posibilita compartir, intercambiar y poner en tensión estos variados saberes y conocimientos que, desde esta mirada, son comprendidos y valorados en su complementariedad (Rodrigues Brandao, 1986).

Por ello, cuando hablamos de "producción de conocimiento conjunto" estamos refiriéndonos a todos los participantes de este proceso en términos de interlocutores, es decir, de personas que, al buscar el diálogo intercultural, orientan sus prácticas a superar las relaciones de poder involucradas en el proceso de producción de conocimiento (Cardoso de Oliveira, 1998; Bartolomé, 2003). De esta forma estamos reconociendo que la intersubjetividad es crucial para la construcción del entendimiento mutuo necesario para resolver las diferencias $y$ elaborar propuestas sólidas que sean satisfactorias para todas las partes (Foreline, 2004).

La redacción del protocolo realizada por miembros del Laboratorio de Investigaciones en Antropología Social y referentes de la
Comunidad Nam Qom, con quienes compartimos la trayectoria de investigación, implicó no solo un compromiso sino un pensar y sentir juntos: antropólogos e indígenas. La emoción ha sido una constante en nuestra trayectoria y entendemos que son varias las razones para que así sea: un marco teórico que no excluye la subjetividad en la producción de conocimiento y que pone énfasis en la dialéctica sujeto cognoscente/sujeto de investigación para dar cuenta de su independencia objetiva e interacción dinámica en el proceso de producción de conocimiento (Schaff, 1992); el largo alcance de la investigación (Braudel, 1970 [1968]), y el hecho de permitir que la existencia del otro nos conmueva, que su dolor nos preocupe, que sus alegrías nos movilicen. En palabras de Miguel Bartolomé (2003):

De allí la solidaridad y el compromiso que suele generarse en un etnógrafo respecto a los pueblos con los que ha convivido y en cuyo mundo afectivo ha tratado o tenido que participar con mayor o menor éxito. Ese compromiso suele desconcertar a quienes ven a las poblaciones indígenas exclusivamente como problemas de investigación, residuos de un pasado o sólo como seres carenciados; "pueblos objeto" alejados de la vida y la experiencia propias. $Y$ es que no sólo nos relacionamos con los otros a través de la razón sino también a través de la afectividad. La propuesta etnográfica es, además de una legítima búsqueda científica, una compleja experiencia afectiva en la que el análisis conceptual no excluye la vivencia personal (p. 219).

Fue así que nos dejamos permear por las demandas y las interpelaciones que los referentes indígenas colocaban en cada encuentro y reflexionamos hacia el interior del equipo respecto de estos procesos, permitiendo incluso que las propias interpelaciones y demandas fueran objeto de reflexión en la búsqueda de construir un conocimiento "plural". 


\section{Desafíos a futuro: sobre "investigadores militantes" e "intelectuales nativos"}

En la provincia de Buenos Aires aún no se ha logrado un reconocimiento pleno de los pueblos indígenas que la habitan $\mathrm{y}$, a pesar de los avances realizados en materia legislativa, sus derechos se ven continua y cotidianamente vulnerados. De allí que continuemos trabajando conjuntamente con el objetivo de contribuir tanto al reconocimiento pleno de la existencia de los pueblos indígenas que habitan el territorio provincial y nacional como para hacer efectivo el cumplimiento de los derechos de estos y sus integrantes. En este contexto elaboramos el "Protocolo de actuación para organismos gubernamentales de la provincia de Buenos Aires que reciben demandas de personas, comunidades y pueblos indígenas por la efectivización de sus derechos" (2017) sintetizando una experiencia compartida con referentes indígenas de más de treinta años. Resultado de lo solicitado por la Defensoría, el trabajo antes presentado constituye un paso inédito en la posibilidad de dejar plasmadas una serie de recomendaciones que, surgidas del diálogo entre la academia y los pueblos indígenas, enriquecen la posibilidad de implementar la legislación vigente y evitar que ciertos obstáculos epistemológicos (Bachelard, 2000 [1948]) obturen la posibilidad de avanzar en el tratamiento de una temática sumamente significativa.

La construcción de lineamientos de análisis teóricos y epistemológicos que referenciamos en este trabajo está vinculada a los planteos de Arturo Escobar (2003) en torno a la descolonización de la subjetividad y la ruptura de la episteme de la colonialidad, desde los cuales señala, como desafío, el poder ser de alguna manera el "otro" sin dejar de ser "uno mismo".
Su propuesta implica reconocer el trabajo pionero de Bonfil Batalla, quien, a fines de la década de 1980, con su obra México profundo. Una civilización negada, refiere la necesidad de acercarse a ese "otro" México, formado por una gran diversidad de pueblos, comunidades y sectores sociales que, en cuanto portadores de singulares visiones de mundo y formas de organizar la vida, tienen su origen en la civilización mesoamericana. El autor habla de esta "civilización negada", cuya presencia, afirma, es imprescindible reconocer para que, al develar prejuicios y al liberar nuestro pensamiento colonizado, podamos explorar la posibilidad de imaginar, proyectar y materializar otras lógicas.

Son estas ideas las que, en los últimos años, condujeron a aceptar formalmente tesis como la de la "ciencia valorativa", que supone considerar la dialéctica subjetividad/objetividad; a resaltar la función del compromiso en la producción científica, y a dar lugar a la visibilización de cientííicos "rebeldes" que ponen a prueba la estructura tradicional de sus disciplinas trabajando/militando en diferentes formas por el cambio estructural de sus sociedades como parte vital de su tarea, sin abdicar por ello del rigor científico ni desconocer las demandas del medio académico (Fals Borda, 2013). El rigor es asequible y las demandas factibles de responder a través de la vigilancia epistemológica, de la explicitación de las condiciones contextuales, históricas y personales, y de la realización de la investigación.

Con frecuencia no solo investigamos, escribimos o hablamos "con" o "junto a" comunidades y referentes indígenas, sino "para" ellos. En los inicios disciplinares los antropólogos llevaban ropa, cuchillos, tabaco, medicamentos y otros objetos como forma de "pago" por entre- 
vistas, fotografías y filmaciones. En la actualidad se han transformado los términos de las negociaciones necesarias para el desarrollo de las investigaciones antropológicas y la permanencia de los académicos en el campo. Quienes otrora fueran considerados "objetos" pasivos de nuestra disciplina, meros "instrumentos" a ser manipulados dentro de las técnicas de recolección de información y construcción de datos, en la actualidad discuten con nosotros los términos para la realización de las investigaciones y somos los antropólogos quienes pasamos a servir como mediadores, asesores y técnicos en diferentes situaciones, como la elaboración y la ejecución de proyectos colectivos-comunitarios, la realización de peritajes, la participación en las definiciones de políticas públicas y de regularización dominial, entre otras labores posibles en nuestro campo de formación.

En el año 2011, un referente indígena qom expresaba esta situación de la siguiente manera: "Ya no es más como antes... que el antropólogo preguntaba: ¿Qué comés? ¿Cómo dormís? Y el indígena contestaba mostrándole los dientes [con una sonrisa]... Usted tiene sus intereses y yo tengo los míos... parece que el toba se retoba".

Actualmente los sujetos que forman parte de objetos de investigación reivindican relaciones equitativas para su participación en las investigaciones, en la medida que estas constituyen parte de proyectos sociales que las exceden. Entre tanto, un cierto "malestar" al interior de la disciplina impulsa reflexiones orientadas al análisis de la participación de antropólogas(os) y nativas(os) en la producción etnográfica (Pacheco de Oliveira, 2006). Estos cuestionamientos están dirigidos a dar lugar a un tipo de relación social diferente entre las personas involucradas en el proceso de investigación, a alcanzar una interlocución equilibrada. Para lograrla "son necesarias una actitud ética y una conducta personal orientadas por el respeto mutuo y por el valor del diálogo, que sólo resultan factibles de ser construidas a partir de la amistad y la confianza" (Bartolomé, 2003, p. 210).

No es necesario renunciar a la empatía y a la afectividad para traducir un hecho biográfico en un dato científico. Son estos sentimientos los que posibilitan, con frecuencia, la investigación y generan solidaridad y compromiso respecto de los pueblos con los que las(os) antropólogas(os) conviven y trabajan. Como dijera Miguel Bartolomé (2003) en la cita antes referenciada: "Ese compromiso suele desconcertar a quienes ven a las poblaciones indígenas exclusivamente como problemas de investigación, residuos de un pasado o sólo como seres carenciados; 'pueblos objeto' alejados de la vida y la experiencia propias" (p. 219).

Este compromiso suele despertar sospechas, como aquella que señala que la dimensión política asumida por el trabajo de los antropólogos en "el campo" estaría menoscabando su carácter científico. La persistencia de esta suposición se funda en lo que Pacheco de Oliveira (2006) Ilama "verdades disciplinares operacionales", una de las cuales es la de suponer la "externalidad de la mirada antropológica”, preocupada fundamentalmente por alcanzar la objetividad. Al respecto, Fals Borda (2013) señala:

A diferencia del observador naturalista, se sabe que en las disciplinas sociales el observador forma parte del universo por observar. Esta condición especial había sido oscurecida por los cánones positivistas sobre la "objetividad" y la "neutralidad" en la ciencia, con la consecuencia de que algunas técnicas de campo como la "observación participante" y la "observación por experimentación" (muy conocida entre antropólogos) 
tendían a conservar las diferencias entre el observador y lo observado (p. 218).

La pretensión de desconocer la responsabilidad sociopolítica del antropólogo en la producción de conocimiento, al pensar este proceso sin problematizar la categoría de poder, ha sido históricamente cuestionada por la antropología crítica latinoamericana, que ha dado cuenta de las múltiples compulsiones a las que los pueblos indígenas han sido sometidos, problematizando sus respuestas y resistencias, acuñando conceptos que no refieren a la lejanía, sino que dan cuenta de la proximidad sociopolítica entre investigadores y sujetos que forman parte del objeto de investigación.

Sin embargo, persiste la sospecha de que la dimensión política asumida en el trabajo de campo estaría menoscabando el carácter científico de la disciplina, de la misma manera en que se mantiene también la consideración de que la dimensión de productores de conocimiento asumida por los referentes indígenas en las universidades estaría perjudicando el "pacto académico" (Maidana et al., 2018). Ello encuentra su origen en la estructura de poder de la colonialidad en la que se enraiza el modo de percibir y objetivar a los "otros" -como "meros informantes"-, y que reserva solo a los antropólogos el ejercicio de la observación y el análisis. Como señala el mismo Pacheco de Oliveira (2006):

En los antiguos moldes en que fue concebido, el encuentro entre el "antropólogo" (siempre pensado como el "de afuera") y el "nativo" sólo ocurre por estar enmarcado en un cuadro colonial mayor, que prescribe conductas y representaciones diversas para cada uno de estos actores sociales, haciendo posible que se instaure una relación unilateral de investigación y producción de conocimiento (p. 206).
A pesar de, y debido a las sospechas que aún genera el marco teórico / epistemológico que guió la redacción del Protocolo, proponemos seguir trabajando en este sentido. En función de ello, en febrero de 2018, reconociendo la significación del trabajo encomendado a nuestro equipo de investigación, presentamos formalmente a la Defensoría del Pueblo de la provincia de Buenos Aires una sugerencia de conformación de una mesa de diálogo intercultural para continuar profundizando la reflexión sobre políticas públicas referidas a pueblos indígenas. Sugerimos que esta fuera integrada por representantes de la Defensoría del Pueblo de la provincia de Buenos Aires, del Laboratorio de Investigaciones en Antropología Social (LIAS) y del equipo responsable de redacción de este protocolo; de la Comisión de Trabajos Interculturales con Pueblos Indígenas y Migrantes de la Secretaría de Extensión de la Facultad de Trabajo Social (FTS) de UNLP, con la que articulamos; de los pueblos indígenas de la provincia de Buenos Aires a través del Consejo Indígena de la Provincia de Buenos Aires $(\mathrm{CIBA})^{19}$, compuesto por ocho representantes pertenecientes a los pueblos qom, guaraní, mapuche-tehuelche y kolla, así como de aquellas instituciones que la Defensoría del Pueblo de la provincia de Buenos Aires considerara pertinente.

Esta propuesta se funda en la importancia que el tratamiento de la cuestión indígena implica, no solo en términos de reparación histórica, sino también para la comprensión de la cuestión nacional y, por lo tanto, para la construcción de una sociedad más justa. 


\section{Consideraciones finales}

Al referir al "Protocolo de actuación para organismos gubernamentales de la provincia de Buenos Aires que reciben demandas de personas, comunidades y pueblos indígenas por la efectivización de sus derechos" y su proceso de gestación planteamos una postura teórico-metodológica que reconoce que quienes participan del proceso de producción de conocimiento interactúan de manera compleja, discutiendo intereses, fijando responsabilidades y elaborando argumentaciones. De esta forma nos apartamos de la manera tradicional de distinguir entre "gentes observadas" y "obser- vadores" de los procesos en análisis. Reconocemos luego que tanto los "unos" como los "otros" trabajan de manera conjunta y producen "conocimiento conjunto", pues todos son sujetos pensantes y actuantes dentro de la labor investigativa y aportan a la construcción de un conocimiento que tanto por su origen como por sus características categorizamos como "plural". Desde esta postura, y ante las sospechas que aún genera, proponemos seguir trabajando para que la antropología, desde su dimensión de herramienta crítica de comprensión y cambio social, no solo aumente su capacidad explicativa, sino también, y al unísono, su capacidad de acción constructora de un futuro mejor.

\section{Notas}

${ }^{1}$ El denominado Censo Indígena Nacional (CIN), instituido por el Decreto № 3998 del 27 de mayo de 1965 desde el Ministerio del Interior, constituye un importante antecedente de medición de la población indígena a nivel nacional, en un contexto sociopolítico y económico claramente diferente del de la sociedad blanca decimonónica. Sobre las lógicas clasificatorias que guiaron la elaboración del mismo, su realización e impacto ver Maidana et al. (2010).

${ }^{2}$ Atacama, ava guaraní, aymara, chané, charrúa, chorote, chulupi, comechingón, diaguita-calchaquí, guaraní, huarpe, kolla, lule, maimará, mapuche, mbyá guaraní, mocoví, omaguaca, ona, pampa, pilagá, quechua, rankulche, sanavirón, tapiete, tehuelche, qom, tonocote, tupí guaraní, vilela y wichí.

${ }^{3}$ Ciudad Autónoma de Buenos Aires (CABA) y 24 partidos de Gran Buenos Aires (GBA).

${ }^{4}$ Actualizado el 30 de noviembre de 2018. Recuperado de http:// datos.jus.gob.ar/dataset/listado-de-comunidades-indigenas

${ }^{5}$ Los registros de las personerías jurídicas pueden ser a nivel nacional -bajo la forma de comunidad indígena- en el Registro Nacional de Comunidades Indígenas (Re.Na.Ci) del INAI, o provincial -ya sea bajo la forma de comunidad indígena u otras formas organizativas, tales como asociaciones civiles, agrupaciones comunitarias, etc.-.

${ }^{6}$ El Relevamiento Territorial de Comunidades Indígenas fue ordenado por la Ley Nacional № 26.160 y reglamentado por Decreto № 1122/07 del Poder Ejecutivo Nacional. Mediante este se facultó al INAI para aprobar "los programas que fueren menester para la correcta implementación del relevamiento técnico-jurídicocatastral de la situación dominial de las tierras ocupadas por las comunidades indígenas originarias del país, para la instrumentación del reconocimiento constitucional de la posesión y propiedad comunitaria". En consecuencia, esta institución procedió al dictado de la Resolución № 587/07, a través de la cual se creó el Programa Nacional Relevamiento Territorial de Comunidades Indígenas (Re. Te.C.I.) -ejecución de la Ley 26.160-. Los objetivos y la metodología de este programa se pueden consultar en el documento recuperado de http://www.desarrollosocial.gob.ar/biblioteca/relevamiento-territorialde-comunidades-indigenas/

${ }^{7}$ Relativo a Buenos Aires, una de las 23 provincias que junto con la ciudad autónoma homónima conforman la República Argentina.

${ }^{8}$ La Defensoría es un organismo de derechos humanos, autónomo e independiente, que promueve los derechos individuales y colectivos de los habitantes de la provincia de Buenos Aires y de quienes se encuentren en tránsito por ella. Controla también a las instituciones y funcionarios(as) de gobierno para el cumplimiento de sus deberes y el respeto de la Constitución y las leyes (Artículo 86 de la Constitución Nacional, Artículo 55 de la Constitución Provincial).

${ }^{9}$ Fundado en 1995, el LIAS pertenece a la Facultad de Ciencias Naturales y Museo (FCNyM) de la Universidad Nacional de La Plata (UNLP). En el marco del mismo se promueven y desarrollan proyectos de investigación/extensión universitaria tendientes a analizar las problemáticas socioculturales que caracterizan a la ciudad de La Plata -capital de la provincia de Buenos Aires-y sus alrededores, así como otras regiones del país que requieren investigaciones 
que analicen los aspectos étnicos presentes en temáticas tales como procesos migratorios, acceso a la tierra, movimientos por la ciudadanía y educación intercultural, entre otros. Las dos principales líneas de investigación son: "Identidad/etnicidad/interculturalidad. Indígenas en ciudad" y "Etnografía de procesos educativos escolares en los distintos niveles formales. Prácticas escolares/escolarización/ educación indígena".

${ }^{10}$ Las primeras familias toba-qom (el pueblo con mayor presencia en la provincia de Buenos Aires) llegaron a la periferia de la ciudad de La Plata en 1991. Con la formalización de su organización en torno a la asociación civil Ntaunaq Nam Qom, actualmente Comunidad Nam Qom, lograron la autoconstrucción de 36 viviendas familiares que fueron finalizadas en 1996. La dinámica colectivo/comunitaria se expresó en dicho proceso y está presente hoy en un sinnúmero de proyectos educativos, laborales y de mejoramiento del entorno. Esta dinámica, así como las condiciones estructurales que originan migraciones hacia las periferias de las grandes ciudades, ha sido analizada en conjunto con los investigadores del LIAS. Dichos análisis han derivado en exposiciones conjuntas en congresos y jornadas de investigación y extensión universitaria, así como en la publicación de artículos científico-académicos en coautoría.

${ }^{11}$ Recuperado de https://www.defensorba.org.ar/contenido/ protocolo-de-actuaci

${ }^{12}$ En términos de Magdalena Gómez Rivera (1993) quien, con el término "verdad legal/judicial" refiere al relato que se construye en relación con las instituciones judiciales y la legislación vigente, muchas veces al margen de la verdad real o aun, en ocasiones, en su contra. Se entiende por verdad real el escenario étnico y social donde se desarrollan los hechos que son materia de litigio $y$, en general, todo el universo de valores culturales propios de los pueblos indígenas.

${ }^{13}$ Este reviste singular relevancia ya que, aprobado por la Ley 24.071/92, es el único instrumento normativo obligatorio específico en el tema, pues, como señala Magdalena Gómez Rivera (2018), en la Organización de Naciones Unidas (ONU) se aprobó la Declaración sobre los Derechos de los Pueblos Indígenas en 2007 sin fuerza vinculante y lo mismo sucedió en 2016 con la adopción, en el seno de la Organización de Estados Americanos (OEA), de la Declaración Americana sobre los Derechos de los Pueblos Indígenas, cuyo contenido es aspiracional.

${ }^{14}$ El panel "Indígenas y políticas sociales. Presencias y reclamos" fue organizado con el objetivo general de trabajar la presencia efectiva de gente indígena en las actividades antropológicas. Constituido por representantes indígenas vinculados a trabajos antropológicos de investigación y acompañamiento/asesoramiento, dicho espacio fue coordinado por la doctora Liliana Tamagno y la profesora Virginia Ceirano (UNLP-Argentina) y contó con la presencia de los profesores João Pacheco de Oliveira (UFRJ-Brasil) y Oscar Agüero (UFRGSBrasil) y la doctora Nora Arias (UNR-Argentina) como invitados especiales. Durante el desarrollo del mismo, los testimonios de situaciones particulares, aportados por los referentes indígenas, fueron acompañados por importantes reflexiones sobre la relación academia-pueblos indígenas y por profundos planteos sobre la necesidad de avanzar en el diálogo intercultural.

${ }^{15}$ En el GT 47 "Patrimonio cultural y pueblos indígenas. El trabajo etnográfico en la gestión de propuestas de desarrollo territorial y con identidad" y el Foro 4 "Voces y conocimientos amerindios contemporáneos. Patrimonio cultural y pueblos indígenas en el sur del mundo", coordinados por los doctores María Amalia Ibáñez Caselli (UARM-Perú), Carolina Maidana (UNLP/UNQ-Argentina) y Walmir Pereira (UNISINOS-Brasil), referentes indígenas y antropólogos debatimos sobre los procesos de activación y gestión del patrimonio cultural y sobre los derechos de los pueblos indígenas en estos procesos. En este contexto elaboramos una nota que fue leída en la asamblea final del encuentro, donde señalamos nuestra profunda preocupación por las situaciones de negación, descalificación y violencia que atraviesan los pueblos indígenas en los Estados del MERCOSUR y la necesidad de generar espacios donde los antropólogos no hablen "por" los pueblos indígenas sino "con" los pueblos indígenas y ante sus demandas.

${ }^{16}$ Prorrogada en los años 2009 (Ley 26554), 2013 (Ley 26894) y 2017 (Ley 27400).

${ }^{17}$ Trayectoria de trabajo que se inició en la década de 1980 con el acompañamiento de la búsqueda de un conjunto de familias tobas (qom) de "un pedacito de tierra para vivir juntos en la ciudad y no perder nuestra lengua y nuestra cultura". Ver Tamagno (1986).

${ }^{18}$ Una descripción de diferentes momentos de producción conjunta de conocimiento puede consultarse en Tamagno, Gómez \& Maidana (2012).

${ }^{19}$ EI CIBA integra, junto a cuatro funcionarios del estado provincial vinculados al Consejo Provincial de Asuntos Indígenas (CPAI), un órgano de codecisión perteneciente a la Secretaría de Derechos Humanos de la provincia de Buenos Aires. Su objetivo es cumplir, fomentar y gestionar políticas públicas destinadas a la población indígena radicada en el actual territorio bonaerense, protegiendo, respetando y garantizando los derechos humanos consagrados por la legislación provincial, nacional e internacional. 


\section{Referencias bibliográficas}

Bachelard, G. (2000 [1948]). La formación del espiritu científico. Contribución a un psicoanálisis del conocimiento objetivo. México: Siglo XXI.

Bartolomé, M. (2003). En defensa de la etnografía. El papel contemporáneo de la investigación intercultural. Revista de Antropología Social, (12), 199-222.

Bonfil Batalla, G. (1989). México profundo: una civilización negada. México: Grijalbo.

Bourdieu, P., Chamboredon, J. C. \& Passeron, J. C. (1975) El oficio del sociólogo. México: Siglo XXI.

Braudel, F. (1970 [1968]). La historia y las ciencias sociales. Madrid: Alianza.

Cardoso de Oliveira, R. (1998). O trabalho do antropólogo. São Paulo: Paralelo 15, Universidade Estadual Paulista.

Cohen, A. (1974). Urban Etnicty. London: Tabistok Publications

Escobar, A. (2003). Mundos y conocimientos de otro modo. El programa de investigación de modernidad/colonialidad latinoamericano. Tabula Rasa, (1), 51-86.

(2014). Sentipensar con la tierra: nuevas lecturas sobre desarrollo, territorio y diferencia. Medellín: Universidad Autónoma Latinoamericana (UNAULA).

Fals Borda, O. (2013). Ciencia, compromiso y cambio social. Buenos Aires: El Colectivo.

Foreline, L. (2004). Applying Anthropology in Brazil: Professionalism and the Commitment to Social Action. Practicing Anthropology, 26(3), 2-4

Gómez Rivera, M. (1993). Sobre la naturaleza del derecho indígena: reconocimientos constitucional y legales. Alteridades, 3(6), 87-100.

(2018). Lectura comentada del Convenio 169 de la Organización Internacional del Trabajo. México: Suprema Corte de Justicia de la Nación-Dirección General de Estudios, Promoción y Desarrollo de los Derechos Humanos.

INDEC (2006). Encuesta Complementaria de Pueblos Indígenas: resultados provisionales 2004-2005. Buenos Aires: Instituto Nacional de Estadísticas y Censos.

Lima, A. C. de S. (2012). Los nativos y sus antropólogos. En De Souza, J. \& Maidana, C. (Comps.), Antropología de los nativos. Estrategias sociales de los sujetos en la investigación (pp. 6-11). La Plata: Editorial de la Universidad de La Plata (EDULP). Recuperado de http://www.bfa.fcnym.unlp.edu.ar/catalogo/doc_num. php?explnum_id=11

Maidana, C., Tamagno, L. \& Martínez, A. (2018). Pueblos indígenas y academia. Sobre la gestación de espacios de producción conjunta de conocimiento. En Congreso de Historia de la Antropología Argentina, Simposio 6: Los Pueblos Indígenas y la Antropología Argentina. Masotta, C. \& Scheinsohn, V. (Coords.). Instituto Nacional de Antropología, Buenos Aires.

Maidana, C., Zubrzycki, B., Samprón, A., Di Socio, J. M., García, S., Colangelo, A., Gómez, J. \& Tamagno, L. (2010). Censos y pueblos indígenas en Argentina. Cuadernos de Antropología, 5(2), 33-52.

Maidana, C., Zubrzycki, B. \& Ottenheimer A. C. (2014). Estado y tratamiento de la diversidad etnoracial. Los dispositivos de relevamiento de población indígena y afrodescendiente. En Tamagno, L. \& Maffia, M. (Coords.), Indígenas, africanos y afrodescendientes en la Argentina. Convergencias, divergencias y desafíos (pp. 229244). Buenos Aires: Biblos.

Mato, D. (2008). No hay saber "universal", la colaboración intercultural es imprescindible. Alteridades, 18(35), 101-116.

Pacheco de Oliveira, J (2006). Pluralizando tradiciones etnográficas: sobre un cierto malestar en la Antropología. En: Pacheco de Oliveira (Comp.) Hacia una antropología del indigenismo (pp. 201-218). Rio de Janeiro, Lima: Contra Capa.

Rodrigues Brandao, C. (1986). Encuentro de instituciones uruguayas con Carlos Rodrigues Brandao. En Fals Borda, O. \& Rodrigues Brandao, C. (Eds.), Investigación participativa. Montevideo: Instituto del Hombre, Ediciones de la Banda Oriental.

Schaff, A. (1992). Historia y verdad. México: Grijalbo.

Stavenhagen, R. (2007) Informe presentado al Consejo de Derechos Humanos A/HRC/4/32

Tamagno, L. (1986). Una comunidad toba en el Gran Buenos Aires: su articulación social. En Actas del II Congreso Argentino de Antropología Social.

(1991). La cuestión indígena en la Argentina y los censores de la indianidad. América Indígena, $L /(1), 123-152$.

Tamagno, L., García, S. M., Ibáñez Caselli, M. A., García, M. C., Maidana, C., Alaniz, M. \& Solari Paz, V. (2005). Testigos y protagonistas: un proceso de construcción de conocimiento conjunto con vecinos Qom. Una forma de hacer investigación y extensión universitaria. Revista Argentina de Sociología, 3(5), 206-222.

Tamagno, L., Gómez, J. \& Maidana, C. (2012). Los caminos de la investigación. Acerca de verdades y utopías. En De Souza, J. \& Maidana, C. (Comps.), Antropología de los nativos: estrategias sociales de los sujetos en la investigación (pp. 175-184). La Plata: Editorial de la Universidad de La Plata (EDULP). 